\title{
Surface Modification of PAN/PVA Nanofibers by Atmospheric Pressure Air Dielectric Barrier Discharge Plasma for Glucose Oxidase Immobilization
}

Mehdi Jahanfar ( $\nabla$ m_jahanfar@sbu.ac.ir)

Shahid Beheshti University

Saeed Javadi Anaghizi

Shahid Beheshti University

Sepideh Asadi

Shahid Beheshti University

Hamid Ghomi

Shahid Beheshti University

\section{Research Article}

Keywords: nanofiber, enzyme immobilization, atmospheric pressure air dielectric barrier discharge plasma (DBD), reusability, storage stability

Posted Date: January 25th, 2022

DOI: https://doi.org/10.21203/rs.3.rs-1246651/v1

License: (9) This work is licensed under a Creative Commons Attribution 4.0 International License.

Read Full License 


\title{
Surface modification of PAN/PVA nanofibers by atmospheric pressure air dielectric barrier discharge plasma for glucose oxidase immobilization
}

\author{
* Mehdi Jahanfar'1, Saeed Javadi Anaghizi², Sepideh Asadi' ${ }^{1}$, Hamid Ghomi \\ 1. Department of Cell \& Molecular Biology, Faculty of Life Sciences \& Biotechnology, Shahid \\ Beheshti University, Tehran, Iran \\ 2. Central Laboratory Of Shahid Beheshti University, Tehran,Iran \\ 3. Laser and Plasma Research Institute, Shahid Beheshti University, Tehran,Iran \\ *m_jahanfar@sbu.ac.ir
}

\begin{abstract}
Polymeric nanofiber readies an appropriate circumstance for enzyme immobilization for assortment of utilizations. In this examination, we've got made up polyvinyl alcohol /polyacrylonitrile (PVA/PAN) nanofibers utilizing electrospinning. After fabrication of nanofibers, the impact of atmospheric pressure air dielectric barrier discharge plasma (DBD) on PVA/PAN nanofibers were investigated in various times. Among them, 6min treat by air DBD plasma had the most significant impact on glucose oxidase (GOx) immobilization. Attenuated total reflectance-Fourier transform infrared (ATR-FTIR) spectrographic analysis and hydrophilic capacity and contact angle results uncovered that if there should arise an occurrence of DBD plasma modified nanofibers, the carboxyl bounds on a superficial level are expanded. The scanning electron microscopy (SEM) pictures indicated that, after GOx immobilization, the modified nanofibers with plasma has held its nanofiber structure. At last, we examined reusability and storage stability of GOx immobilized on plasma modified and unmodified nanofibers. The results were additional satisfactory for modified nanofibers with relevance to unmodified ones.
\end{abstract}

Key words: nanofiber, enzyme immobilization, atmospheric pressure air dielectric barrier discharge plasma (DBD), reusability, storage stability

\section{Introduction}

The applicability of nanofibers to daily life is undeniable. Nanofibers can be defined as a fiber which are smaller than $1,000 \mathrm{~nm}$ can be considered as nanofibers according to [1,2]. Due to the fact that nanofibers have high surface to volume ratio, porous structure, small pore size, and proper mechanical resistance, 
they have several advantages compared to common textile fibres [3,4]. As the mentioned feature of nanomaterials, they have become a considerable option for using in areas such as filtration $[5,6]$, wound dressing [7], tissue engineering [8], artificial vessels [9], drug delivery systems [10], acoustic materials [11], cosmetics [12], lithium ion batteries [13], distillation [14], and desalination [15] and enzyme immobilization [16]. Among variety of distinctive strategies of polymeric nanofiber generation, electrospinning is additionally favored to other approaches since it has less value and ease among their capability of manufacturing long and ceaseless strands with submicron scale as straightforward as potential. [17]. Actually all nanofiber structures are naturally hydrophobic but depend on existent and number of polar group on nanofibers surface, they attract to water which have considered as hydrophilic nanofibers. Increasing the hydrophilic properties of nanofibers will improve the biocompatibility and permeability of nanofiber layers for water filtration [18], microfiltration [19], seeding or attaching the cells and enzymes on the surface of the layer [20], moisture absorbing [21], the release of proteins [22], and so. According to research, the polymer mixture of polyvinyl alcohol (PVA) and poly acrylonitrile (PAN) polymers in various ratios are used widely in producing the organic fibers by electrospinning, which have combined advantages of both of polymers in the applications. In our recent study have attempted to find the best ratio of PVA and PAN for fabrication [23,24]. In recent decade, biomedicine and biosensors have significant advances and enzyme has since become widely used in these fields. Enzyme efficiency and features of bio-catalyst are greatly dependent on immobilization method and choosing proper substrate for immobilization [25,26]. Numerous researches have centered on various strategies (e.g. cross-linking with aromatic tri-isocyanates, UV or gamma irradiation, etc.) to immobilize enzymes on distinctive supports and have driven to applicable biosensors [27]. In other hand, the efficient immobilization of enzymes on the surface is one of the foremost challenges in developing enzyme-based biosensors. Between distinctive enzymes, glucose oxidase (GOx) is one amongst the main choices for checking the enzymatic behavior on the immobilized substrate [28]. The main known methods for immobilize enzymes are physical adsorption, covalent bonds to water-insoluble functionalized polymers (i.e., organic or inorganic polymers) or entrapment/encapsulation [29]. Among these methods, adsorption is more commercial than other methods because of its simpler and less expensive process, retaining a high catalytic activity, and the possibility of over and over reusing scaffold after inactivation immobilized enzymes which could be considered as the most important advantage of this method [30]. Consequently, enzyme immobilization through adsorption needs a powerful interaction like hydrophobic or ionic interaction between the enzyme and scaffold [31]. Chemical features of the surface, i.e. functional groups on the surface is one of the basic variables in the rate of enzyme absorption by polymer surfaces. Due to demonstrating and enhancing the covalent immobilization of diverse bioactive molecules, appropriate functional group on the surface could be arrange [32]. Accordingly, for expanding the rate of enzyme immobilization, functional groups on polymer surface must be modified. Polymers are almost classified by low surface energy and thus low adhesive properties [33]. Modifying the polymer surfaces to improve physicochemical polymers properties can ensure proper adhesion between polymer and a coating. There are diverse methods to modify polymer surfaces to serve different purposes such as biocompatibility, wettability and functionality for biological applications. These methods can be classified to physical, chemical and mechanical methods. Mechanical methods counting roughening and micromanipulation are not convenient for small changes i.e. under micrometers so they are restricted. Ion implantation [34], electron beam irradiation [35], ultraviolet radiation and gamma-ray irradiation and plasma technology as physical methods have some preference over chemical methods containing oxidize, sulfonate, chlorinate and acetylate. Physical methods are considered as secure and clean processes without chemical-wasting 
[36]. Besides, chemical methods cannot be appropriate for immobilizing biomolecules on the surface of polymers in all cases since having some destructive chemical reactions [37]. Most noticeable dominance which is appointed to plasma treatment is that it causes the reactive moieties to embody onto the surface therefore as to improve the surface energy and reactivity for more functionalization, and consequently enhance the biocompatibility and surface properties while not altering bulk properties [38]. Recently, heaps of methods such as plasma jet, dielectric barrier discharge (DBD), diffuse discharge and gliding arc discharge have been implemented for surface modification by utilizing non-thermal plasmas [39]. Atmospheric pressure plasma modification is one among the appropriate physical strategies which is broadly utilized without using solvent or creating chemical waste including less degradation or alerting polymers bulk properties. Recently DBD plasma-based surface modification has attracted researcher's consideration particularly using of atmospheric pressure air (DBD), which is an efficient and cost-less and commercial strategy [40]. However in present study we would like to increase the immobilization and activity of GOx on PVA/PAN nanofibers manufactured by electrospinning and surface modification of these nanofibers by air-DBD plasma which the advantages have been mentioned before.

\section{MATERIAL \& METHODS}

\section{Materials:}

Polyvinyl alcohol (PVA) with molecular weight of 72,000 and Poly acrylonitrile (PAN) were purchased from Merck Chemical Co. (USA). Malonic acid (MA) and beta-D-glucose was obtained from SigmaAldrich (St Louis, MO, USA). O-dianizidin $\left[\left(\mathrm{CH}_{3} \mathrm{O}\right)\left(\mathrm{H}_{2} \mathrm{~N}\right) \mathrm{C}_{6} \mathrm{H}_{3}\right]_{2}$ with molecular weight of $244.29 \mathrm{~g} / \mathrm{mol}$ was bought from Fluka . Glucose oxidase (GOx) was obtained from Aspergillus niger. (Sigma Prod. Nos. G-6766 ). Horseradish peroxidase (HRP) was acquired from Applichem Co. Dimethyl formamide (DMF) that was utilized as a solvent for polymeric solution, was gotten from Merck Chemical Co. (USA). The buffer utilized in this work is Potassium phosphate $(0.1 \mathrm{M}$, at $\mathrm{pH}$ 6) which was arrenged from sterilized water and Potassium acetate solution.

\section{Fabrication of nanofibers}

Polyvinyl alcohol and Poly acrylonitrile solution (3 Wt. \%) were arranged by dissolving in Dimethyl formamide $\left[\left(\mathrm{CH}_{3}\right)_{2} \mathrm{NC}(\mathrm{O}) \mathrm{H}\right]$, and we utilized malonic acid as a cross linker in polymeric solution and the weight quantitative to mix polyvinyl alcohol with was chosen to be $1: 1$ which were adding to solution simultaneously, then they were stirred for $4 \mathrm{~h}$ via the magnetic stirrer at 80 centigrade till transparent solutions were gotten. Then, were placed in room temperature at stationary conditions for $12 \mathrm{~h}$ as to obtain a uniform solution without bubbles. The schematic of experimental setup is shown in Fig. 1, Polymer solution was loaded to a syringe and then was fixed into a syringe pump with a flow rate of 0.5 $\mathrm{ml} / \mathrm{h} .22 \mathrm{kV}$ of positive voltage was applied to the needle by a high voltage power supply. The distance between needle of syringe to the collection plate of aluminum foil was $15 \mathrm{~cm}$. 


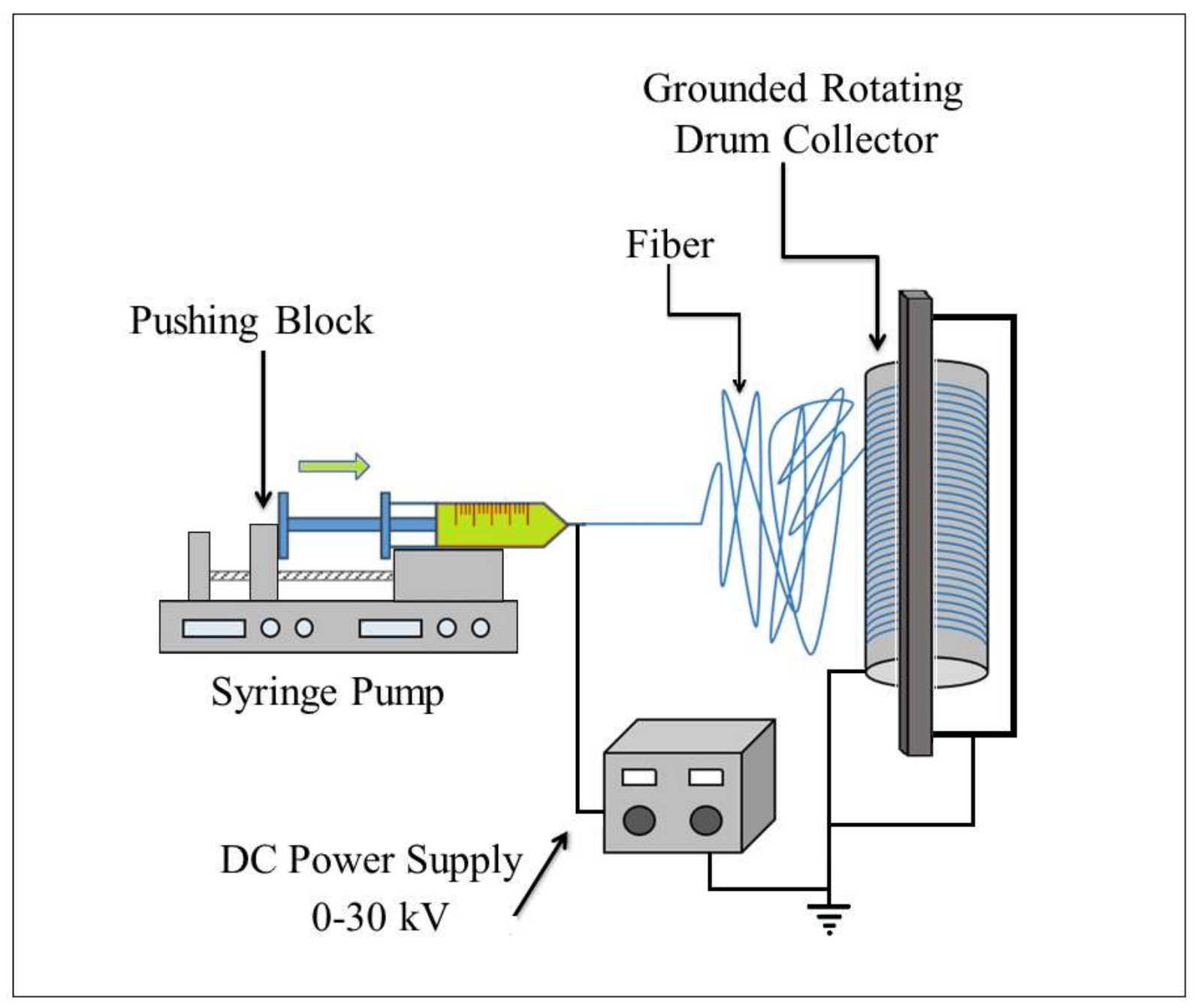

Fig1. Schematic representation of the electrospinning device

\section{Surface modification of electrospun nanofibers by DBD}

The schematic of hand crafted DBD plasma test employed in this paper is appeared in Fig. 2 High voltage electrode comprised of copper rod as a $10 \mathrm{~mm}$ thickness and Teflon's divider tube folded around the high voltage electrode which was covered by $1 \mathrm{~mm}$-thick quartz sheet (>99.9\% SiO2) as dielectric obstruction. A stainless steel mesh was utilized as the second electrode in contact with the quartz which acts as a grounded electrode to produce a homogenous DBD plasma, it is outstanding that no air ought to be available between the mesh and the dielectric in arranged to forestall power loss [41].

The sample was placed on the grounded sample holder at a distance of $2 \mathrm{~mm}$ from the grounded mesh. A half-dozen $\mathrm{kHz}$ DC-pulsed high voltage which is consistence voltage in $11 \mathrm{kV}$ and $0.5 \mathrm{~W} / \mathrm{Cm}^{2}$ discharge power which controlled by lissajous curve was applied between to the electrodes of DBD plasma probe. 
Fig.3 appears the normal voltage and current waveforms for the high voltage conductor with 2-mm slot between the threads samples at $11 \mathrm{kV} V_{P P}$ and $6-\mathrm{kHz}$ frequency.

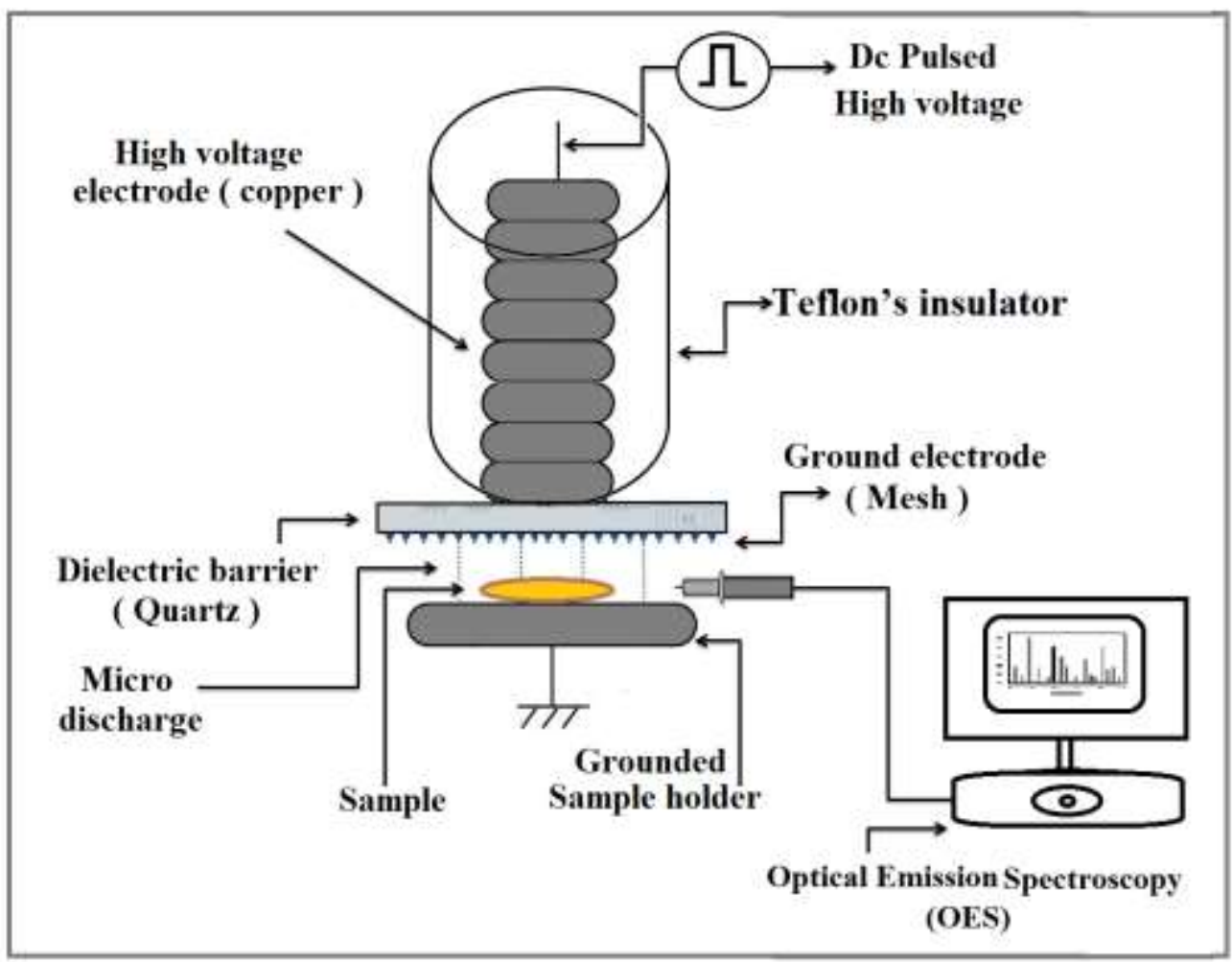

Fig. 2. Schematic of DBD plasma probe 


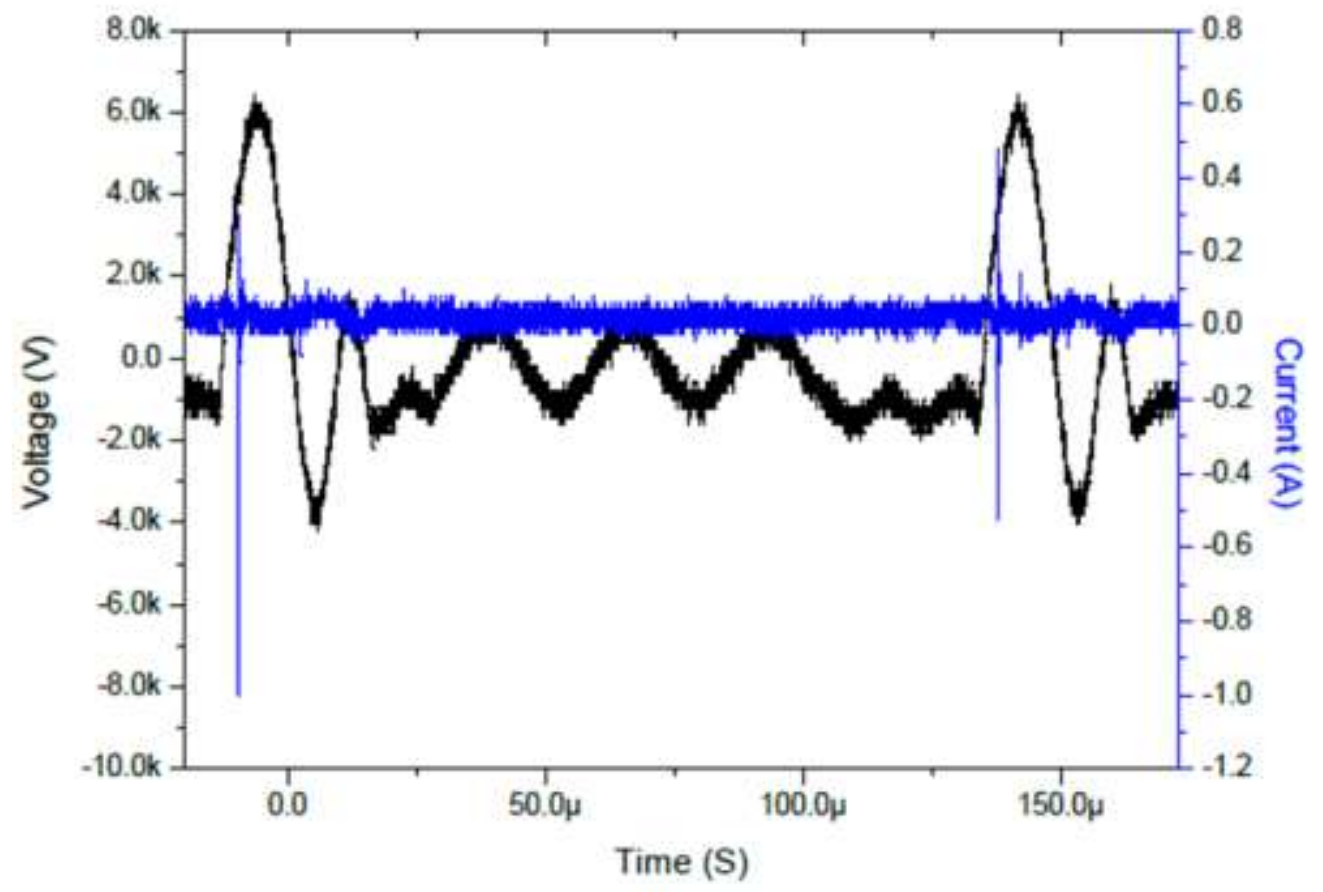

Fig. 3. Current and voltage waveforms of the DBD discharge generated at $11 \mathrm{kV}$ and $0.5 \mathrm{~W} / \mathrm{Cm}^{2}$ discharge power

To examine the plasma arrangement intensity was analyzed by an Optical emission spectroscopy (OES) method utilizing a spectrometer (Ocean Optic HR2000+ES) with the accuracy $0.5 \mathrm{~nm}$.the range of wavelength considered in this investigation is from $200 \mathrm{~nm}$ to $900 \mathrm{~nm}$. [42]. The optic fiber the spectrometer was put agonist of the plasma probe at the same position and condition under the same condition within all tests. When the high voltage applied to the DBD probe comes to the most extreme value, micro discharges form and cross between two electrodes and cause the charges collect on the surface of the quartz-dielectric barrier in several nanoseconds. At the time of few microseconds (the quantity between 2 pulses), the electron releases. At the point of the applied voltage reaches to the next maximum value, micro discharges will begin again [43].

\section{Characterization}

The surface morphology of unmodified and plasma modified nanofibers after immobilization and soak in enzyme buffer solution were discovered with a scanning electron microscope (SEM) (Hitachi SU3500), after $10 \mathrm{~nm}$ thickness gold sputter-coating. We utilized attenuated total reflectance Fourier transform infrared (ATR-FTIR to examine the surface chemical structure of samples, The ATR-FTIR spectra (ATRFTIR-NEXUS 470, THERMO NICOLET CO, USA) of nanofibers were identified in the 4000 to 500 $\mathrm{cm}^{-1}$ wavenumber, with 60 scans at a resolution of $4 \mathrm{~cm}-1$. The topography of nanofibers were resoved an Atomic Force Microscopy (Nano Ink DPN500).

\section{Enzyme immobilization and colorimetric assay}

The surface adsorption method of immobilization is used in this study. Glucose oxidase solutions (with activity 37.4 Unit in phosphate buffer $\mathrm{pH} 7,0.1 \mathrm{M}$ ) were used to spray treated nanofibers for three times. The nanofibers then were cut into tiny pieces $\left(1 \times 1 \mathrm{~cm}^{2}\right)$. The immobilized enzyme's activity was set by 
transferring a small piece of wet nanofiber in the test tube which contained $2 \mathrm{ml}$ phosphate buffer $\mathrm{pH} 7$, $0.1 \mathrm{M}, 100 \mu \mathrm{l}$ of $0.02 \mathrm{mg} / \mathrm{ml} \mathrm{o}$-Dianizidine and $100 \mu \mathrm{l} 18 \%$ glucose solution and $10 \mu \mathrm{l}$ horseradish peroxidase enzyme $(1 \mathrm{mg} / \mathrm{ml})$. Absorbance increase was recorded at $640 \mathrm{~nm}$ every 30 seconds [44].

\section{RESULTS \& DISCUSSION}

\subsection{Optical Emission Spectroscopy (OES)}

Plasma represents a highly efficient environment when in contact with a polymer surface. The primary reactions break the chemical and physical bonds, producing active sites, polar groups and cleavage products onto the polymer surface. Fig. 4 demonstrates that the OES spectra of plasma system. Plasma spectroscopy was utilized to investigate the plasma discharge characteristics. When plasma introduces within the polymer sheet, oxidation cycles will be begun in the plasma probe, surface modification of polymers by a set of reactions between free electrons, ions, radicals, atoms, and molecules which are shaped in plasma. These reactions can be summarized as follows the highly reactive atomic and molecular oxygen species and the very high energy UV photons the surface polarity for various exposed samples, usually demonstrating increase in the surface hydrophilicity and polarity after treatment. These reactions can be summed up next by [45]:

$$
\begin{aligned}
& N_{2}+e \rightarrow N+N+e \\
& N_{2}+e \rightarrow e+N_{2}(A) \\
& N_{2}^{+}+e^{-} \rightarrow 2 N \\
& N^{+}+e^{-} \rightarrow N \\
& O_{2}+O \rightarrow O_{3} \\
& O_{2}+e \rightarrow O+O+e \\
& O_{2}+O \rightarrow O_{3}
\end{aligned}
$$

The emission spectrum of the plasma discharge displayed peaks of $\mathrm{N}_{2}$ second positive band system ( $\left.\mathrm{N}_{2} \mathrm{SPS}\right), \mathrm{N}_{2}{ }^{+}$first negative system $\left(\mathrm{N}_{2} \mathrm{FNS}\right)$ and $\mathrm{O}_{2}$ band system [46]. Table 1 lists the detected peaks of the wavelengths for the $\mathrm{N}_{2}$ SPS systems and the $\mathrm{N}_{2}$ FNS systems and the $\mathrm{O}_{2}$ band system. 
Table 1. The detected peaks of the wavelengths for the $\mathrm{N}_{2}$ second positive band systems and the $\mathrm{O}_{2}$ band system

\begin{tabular}{ccc}
\hline Species (system) & Transitions & Peak positions (nm) \\
\hline $\mathrm{N}_{2}$ second positive band & $C^{3} \Pi \rightarrow B^{3} \Pi$ & $315 ; 337.1 ; 357.7 ; 400$ \\
\hline $\mathrm{N}_{2}{ }^{+}$first negative & $B^{2} \sum_{u}^{+} \rightarrow X^{2} \sum_{g}^{+}$ & $427.8 ; 434.2 ; 441.7$ \\
\hline $\mathrm{O}_{2}$ band & $3 p^{5} P \rightarrow 3 s^{5} S^{0}$ & 777.4 \\
\hline
\end{tabular}

Based on the $\mathrm{N}_{2}$ second positive band system, the excitation of nitrogen molecules within the ground state by direct electron impact is carried out as follows [47]:

$e+N_{2}\left(X^{1} \sum_{g}^{+}\right)_{v=0} \rightarrow N_{2}\left(C^{3} \pi_{u}\right)_{v^{\prime}=0}+e \quad(\Delta \mathrm{E}=11 e V)$

And then, spontaneous radiation of shaped excited state of nitrogen makes $\mathrm{N}_{2}\left(\mathrm{~B}^{3} \pi_{\mathrm{g}}\right)$ as follows [48]:

$N_{2}\left(C^{3} \pi_{u}\right)_{v^{\prime}=0} \rightarrow N_{2}\left(B^{3} \pi_{g}\right)_{v^{\prime \prime}=0}+h v$

Air, which is utilized as carrier gas with humidity of water droplets, ends up to generate of active species and ozone by micro discharge in plasma by means of polymer fibers (M) [49]:

$e+O_{2} \rightarrow e^{+} O\left(3_{P}\right)+O\left(1_{D}\right)$

$O+O_{2}+M \rightarrow O_{3}+M$ 


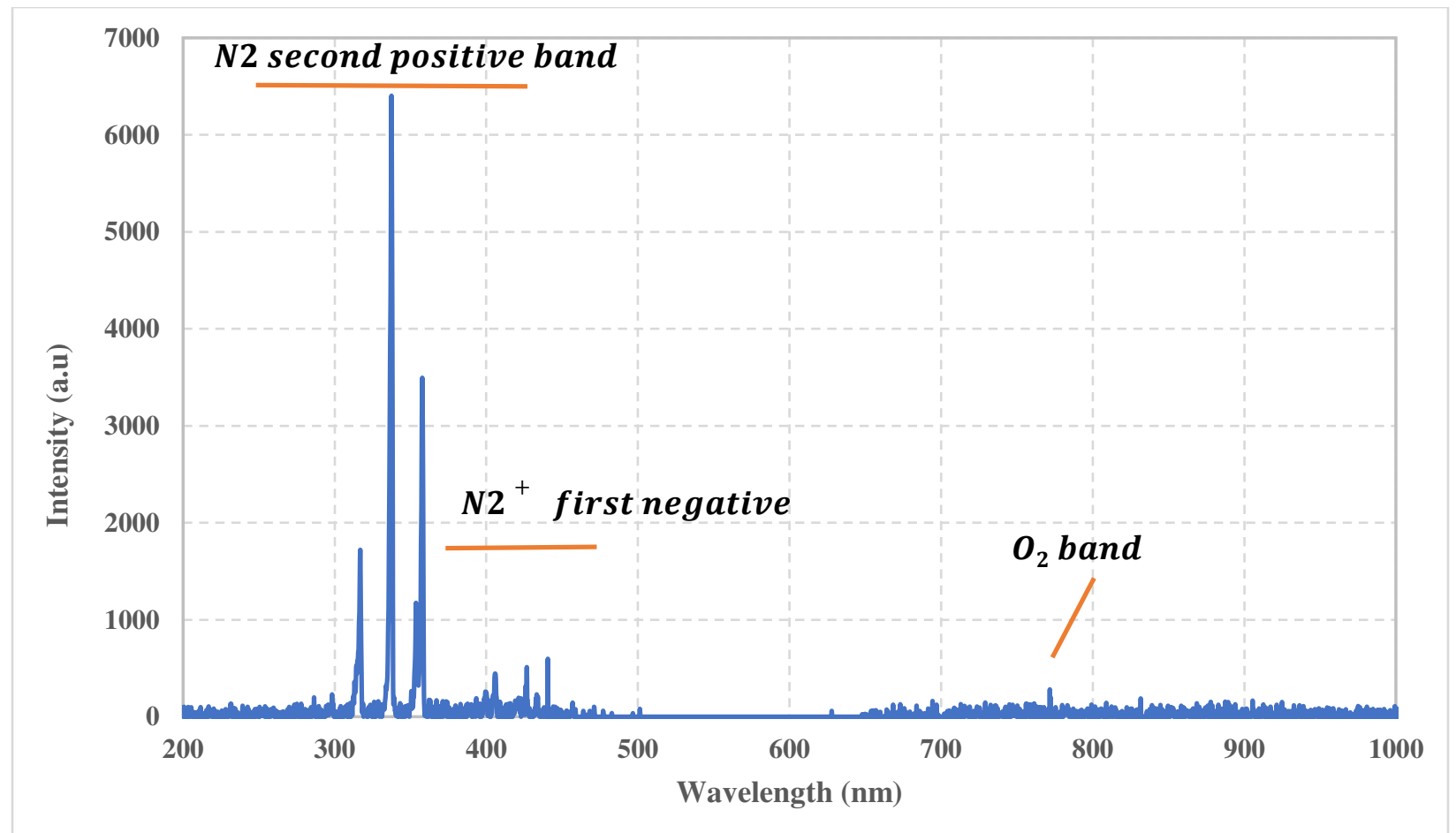

Fig4: emission spectrum of the plasma discharge in plasma probe in $11 \mathrm{kVPP}$ and $6 \mathrm{kHz}$ pulse frequency

The emission spectrum of the plasma discharge in plasma probe has been demonstrated in Fig.4. This spectrum was gotten at $11 \mathrm{kV}_{\mathrm{PP}}$ and $6 \mathrm{kHz}$ pulse frequency. The plasma has occasioned multiple hemolytic bond fissions and ionization occurrences, also secondary collisions. Subsequent molecular fragmentations, reactions, and ionization processes have eventuated in a propagation of functionalities on the plasma-treated or polymerized surfaces high properties of crosslinking and functionalization onto the surface. As illustrated in Fig.4, the higher intensity related to N2 (SPS),(FNS) band and $\mathrm{O}_{2}$ band is 337.43 $\mathrm{nm}, 427.76 \mathrm{~nm}$ and $77.42 \mathrm{~nm}$ respectively.

\subsection{Surface wettability of PVA/PAN nanofiber}

To distinguish the hydrophilicity or hydrophobicity of polymer surfaces often has utilized contact angle measurements [45]. The wettability of PVA/PAN nanofiber before and after plasma excitation were demonstrated by measuring contact angle of water droplets with distinctive disposal times; the water droplet illustration on the surfaces of unmodified and plasma-modified samples are displayed in Fig.5 and the information for them are exhibited in Fig.6. As enhancement plasma exposure time from 0 to 9 minute the contact angles has declined roughly from $126^{\circ}$ to $54^{\circ}$. The enhancement in hydrophilicity inculcates an augment in oxygen-containing groups on the surface of the polymer which can be accomplished in $\mathrm{O}_{2}$ band transaction is due to plasma treatment reactions (11) and (12) [49].
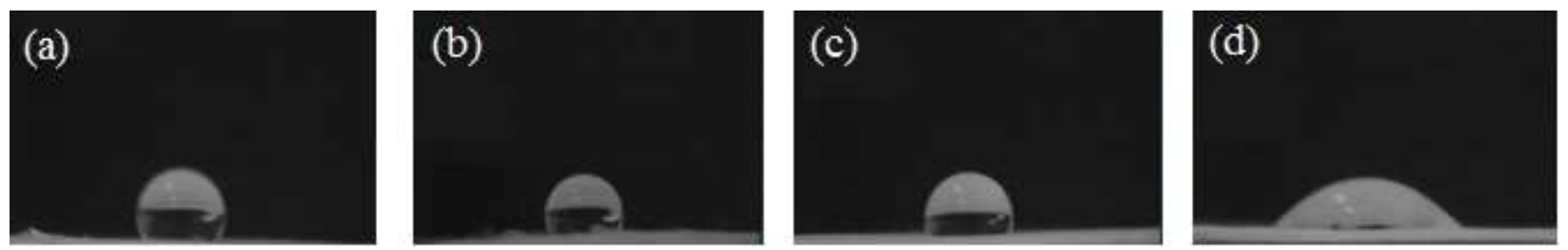
Fig. 5. Water droplet on the surface of PVA/PAN nanofibers (a) unmodified, (b) plasma modified for 3 min, (c) plasma modified for $6 \mathrm{~min}$, (d) plasma modified for $9 \mathrm{~min}$

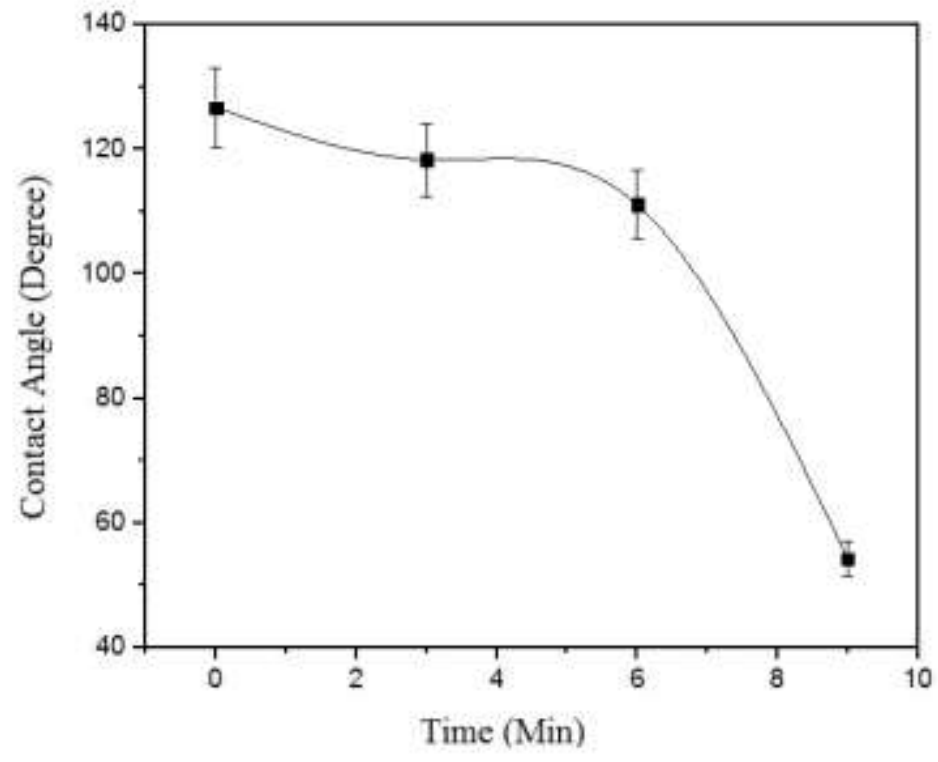

Fig. 6. Water contact angle as a function of plasma treatment time.

\subsection{Surface chemical composition characterization}

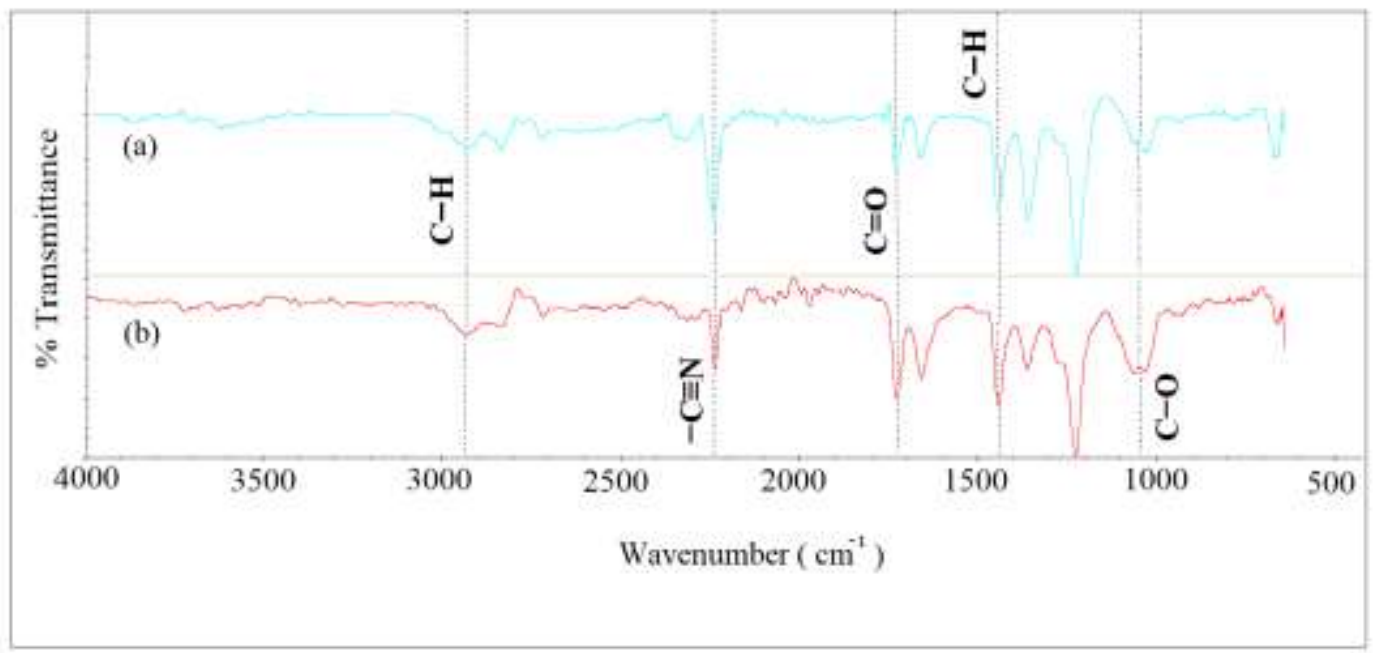

Fig. 7. ATR-FTIR spectra of (a) unmodified, (b) plasma modified PVA/PA nanofiber

ATR-FTIR spectra were obtained from plasma modified and unmodified surfaces to manifest any shift in chemical bonding states on the polymer surfaces. All treatments have been exert in the atmospheric 
pressure at $11 \mathrm{kV}-6 \mathrm{kHz}$. Fig.7 depicted ATR-FTIR spectra of unmodified and plasma modified PVA/PAN nanofibers after 6 minute exposure. The spectrum of both of samples has rendered the emersion of $\mathrm{C}-\mathrm{H}$ stretching at $2920 \mathrm{~cm}^{-1}$ and $\mathrm{C}-\mathrm{H}$ bending band at $1420 \mathrm{~cm}^{-1}, \mathrm{C} \equiv \mathrm{N}$ stretching at 2242 $\mathrm{cm}^{-1}, \mathrm{C}=\mathrm{O}$ at $1736 \mathrm{~cm}^{-1}$ and $\mathrm{C}-\mathrm{O}$ at $1095 \mathrm{~cm}^{-1}$ [41-43]. As can be observed, PVA/PAN nanofibers spectrum which modified by air plasma, has demonstrated higher absorption at $1736 \mathrm{~cm}^{-1}(\mathrm{C}=\mathrm{O}$ stretching bands) and $1095 \mathrm{~cm}^{-1}$ (C-O stretching band) board bands and lower absorption at $2242 \mathrm{~cm}^{-1}$ $\left(\mathrm{C} \equiv \mathrm{N}\right.$ stretch) peaks as compared with unmodified nanofibers. The $\mathrm{C}-\mathrm{H}$ bending band at $2920 \mathrm{~cm}^{-1}$ and $\mathrm{C}-\mathrm{H}$ bending band at $1420 \mathrm{~cm}^{-1}$ has revealed to be either potent [41-43]. The substantial augment in both of the stretching of $\mathrm{C}-\mathrm{O}$ group and the stereomic series in plasma modified scaffold, have demonstrated the effect of plasma on the esterification reaction by activation hydroxyl groups which derived from polyvinyl alcohol throw $\mathrm{N} 2$ second positive band transaction in table 1. The plasma's efficacy on augment the reaction rate of nitrile group to carboxyl group, which is actually done by enhancing the functional group, ultimately has increased the amount of hydrogen bonding with the enzyme in the immobilization phase consequently the activity of immobilized enzymes increased too. Decreased peaks associated with $\mathrm{C} \equiv \mathrm{N}$ bend and increased peaks associated with the bonded carboxylic group are further confirm of this claim. This reaction is referred to free radical formation which produced in plasma procedure.

\subsection{Activity of immobilized enzymes on nanofibers}

The enzyme activity belongs to accessibility of the enzyme active site which is immobilized on the surface of the scaffolds. Moreover, the enzyme activity enhancement can be eventuate of the increase in the amount of enzymes which immobilized on scaffolds. In this experiment the amount of enzyme activity which gauged as follows: $162 \times 10^{-3} \mathrm{U} / \mathrm{cm} 2$ for unmodified nanofibers, $259 \times 10^{-3} \mathrm{U} / \mathrm{cm} 2$ for 3 minutes plasma modified nanofibers, $413 \times 10^{-3} \mathrm{U} / \mathrm{cm} 2$ for 6 min plasma modified nanofibers, $91 \times 10^{-3}$ $\mathrm{U} / \mathrm{cm} 2$ for 9 minutes plasma modified nanofibers. As represented in Fig. 8, in PVA/Pan nanofibers modified by exposing for 6 minutes with air plasma, the activity of enzymes which immobilized on the surface nanofibers has been rather than all other samples. These results have designated better enzyme orientation and immobilization on the 6 minutes plasma modified nanofibers. As compare the amount of enzyme activity between modified and unmodified samples, it can be conclude air plasma has augmented enzyme activity 2.54 times. These conduct can be clarified by the augment in the acid-base group and stereomic $\mathrm{C}=\mathrm{O}$ group which was acquired from ATR-FTIR spectrum. Likewise the less enzyme activity which observed in case 9minutes exposed by air plasma could be associated with light ablation on the polymer surface caused by the increment exposure time of plasma [48]. 


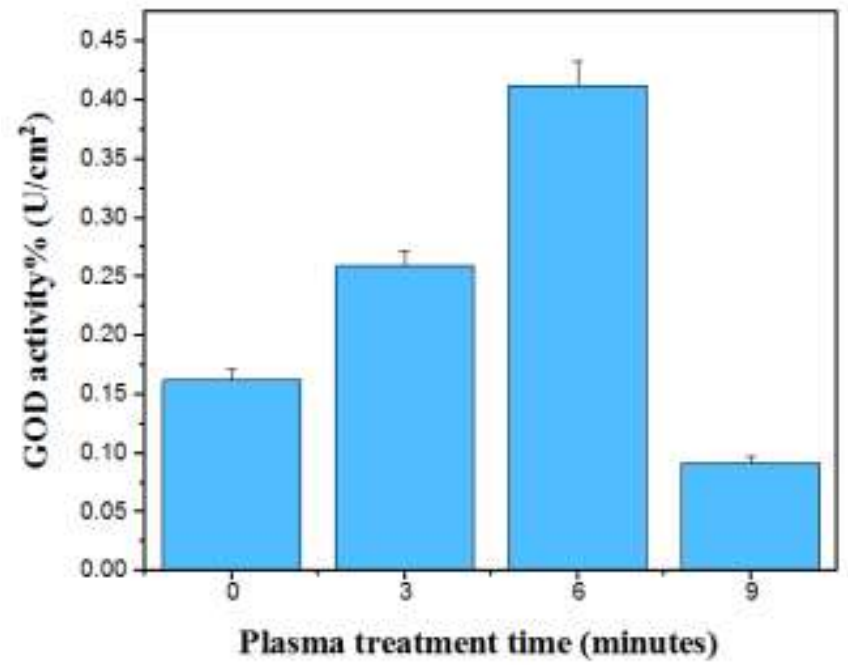

Fig. 8. Activity of immobilized GOD on the surface of plasma modified PVA/PAN nanofibers for three different exposure times.

\subsection{Reusability of immobilized enzymes}

Conforming to the results, it could be conducted the highest enzyme activity has belonged to plasma modified PAN/PVA nanofibers for 6 minutes. Fig. 9 depicts a diagram which graphing reusability of immobilized enzymes on this scaffold throughout a ten consecutive measurements of the enzymatic activity of this nanofiber. As is evident, $71 \%$ of its enzyme activity has maintained after ten times use in 6 min plasma modified nanofibers, whereas the unmodified scaffold have been maintained only $28 \%$ of own activity at their first time use. These outcomes can be inferred from the augment in the amount of immobilized enzymes on the polymer surface owing to the further functional groups formed on the polymer surface after exposure to plasma, as affirmed by ATR-FTIR results.

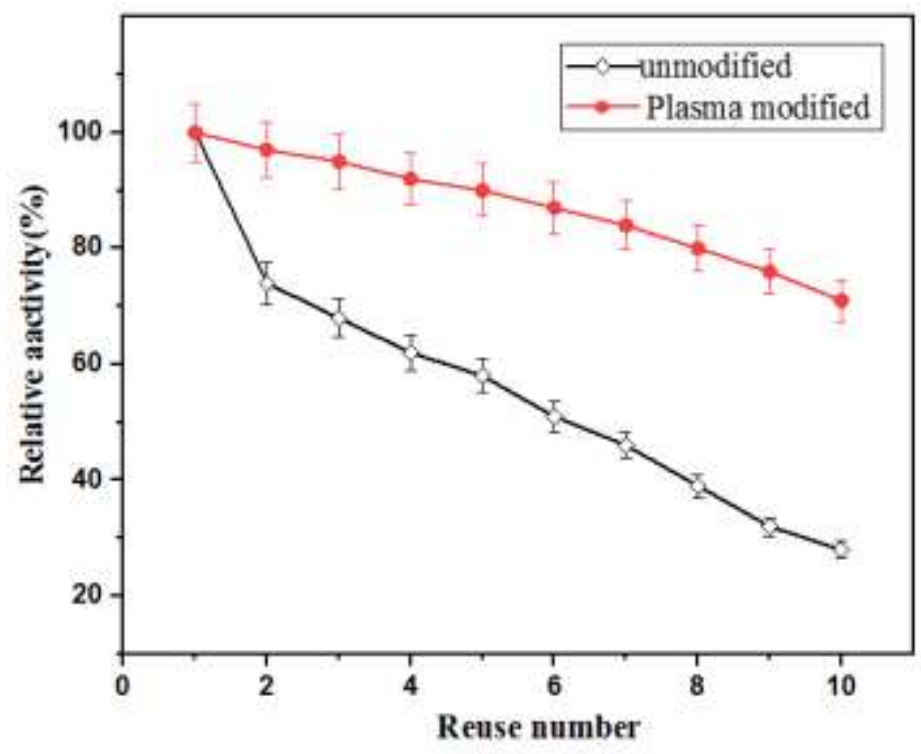


Fig.9 . Reusability of immobilized enzymes on plasma modified PAN/PVA nanofibers for 6 min compared with unmodified PAN/PVA nanofibers.

\subsection{Nanofibers morphology}

Fig.10 has illustrated the scanning electron microscope images of unmodified and air plasma modified PVA/PAN nanofibers for various durations after enzyme immobilization. .It can be acquired from Fig 11, which the diameter of plasma modified nanofibers figured out with image $\mathrm{J}$, has augmented besides 9 min plasma-exposed. It can be concluded by enhancement the exposure time of plasma from 3 to 6 minutes, nanofibers diameter has also increased. This might be related to the fact that more cross-links have been made between polymer chains caused by inducing some chemical modifications such ozone formation and its related transactions. Whereas by more increasing the plasma exposure time up to 9 minutes, a significant decrease in nanofibers diameter has been observed. This observation can be attributed to the light ablation of nanofibers surface which resulting from plasma [44]. Fig. 12 has displayed the SEM images of unmodified and PVA/PAN nanofibers-air plasma modified during various times after drenching in enzyme solution for 60 minutes. The images has indicated that unmodified nanofibers after drenching have lost their nanofiber structure, while plasma modified samples have retained their fiberlike structure. In addition to there has been not significant damage observed in fibers structure. The process of maintaining the fiber-form structure was similar to what has been observed in nanofiber diameter as a result of exposure to air plasma at different times.

a)

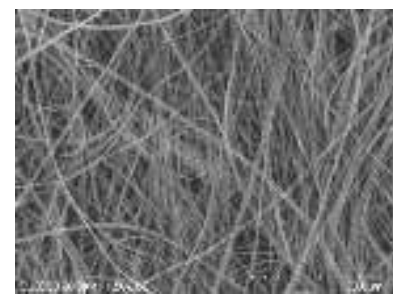

b)

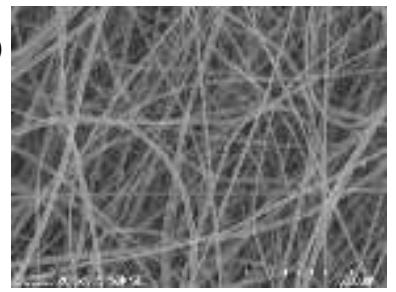

c)

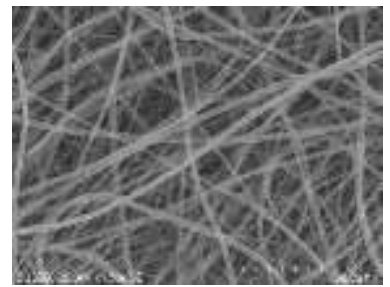

d)

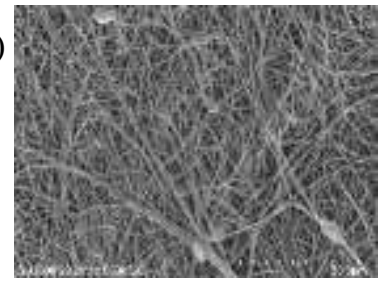

Fig.10 . Electrospun nanofibers PVA/PAN morphology using SEM, (a) unmodified, (b) plasma modified (3min), (c) plasma modified (6min), (d) plasma modified (9min), 


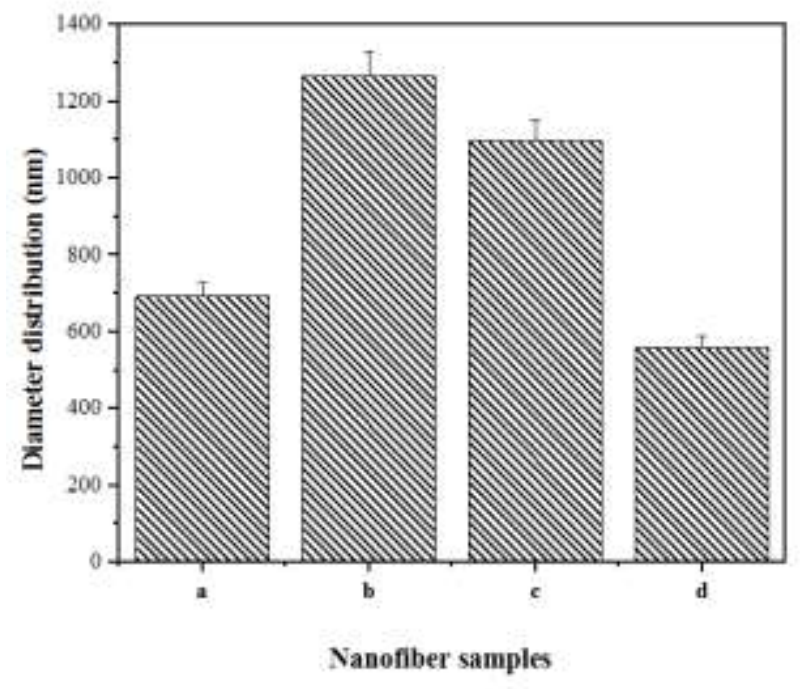

Fig.11 . Diameter distributions of electrospun nanofibers PVA/PAN (a) unmodified, (b) plasma modified (3min), (c) plasma modified (6min), (d) plasma modified (9min),

a)

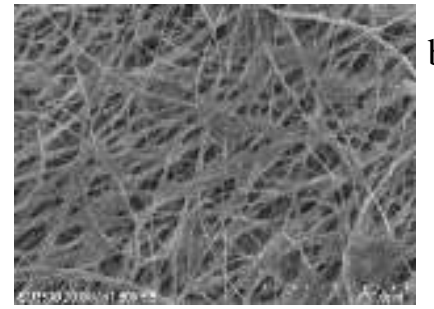

b)

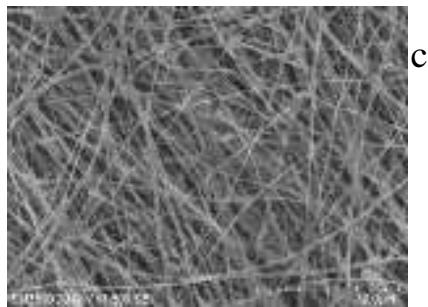

c)

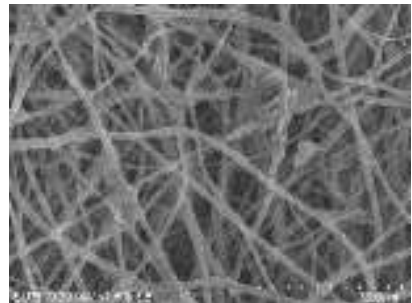

d)

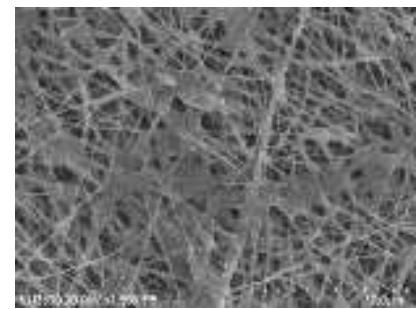

Fig.12. PVA/PAN nanofibers morphology using SEM after drenching in enzyme buffer for 60 minutes, (a) unmodified, (b) plasma modified (3min), (c) plasma modified (6min), (d) plasma modified (9min)

Fig. 13(a) has depicted the AFM image obtained from unmodified PVA/PAN nanofibers and Fig. 13(b) the modified PVA/PAN nanofibers via air plasma during 6minutes. As assumed likewise has been clear from the picture, the surface roughness of PVA/PAN nanofibers by exposing to air plasma, has increased. The outcomes acquired from analyzing the AFM images indicated that, the mean surface roughness (Sa) value of $4.1 \mathrm{~nm}$ in the case of unmodified nanofibers and the value of $7.5 \mathrm{~nm}$ for plasma modified nanofiber has recorded. Values of $6.1 \mathrm{~nm}$ and $9.8 \mathrm{~nm}$ have gauged as the root mean square roughness (Sq) of unmodified and modified plasma nanofibers, respectively.

(a)

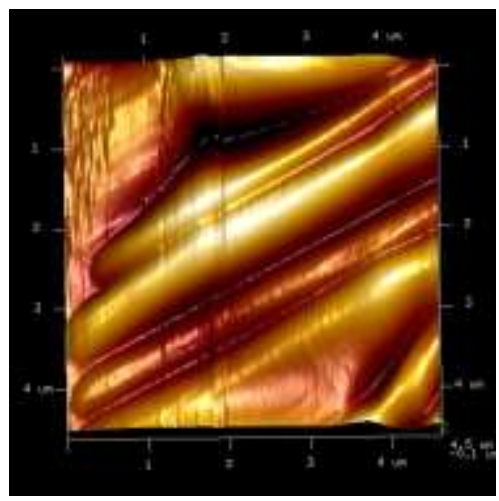

(b)

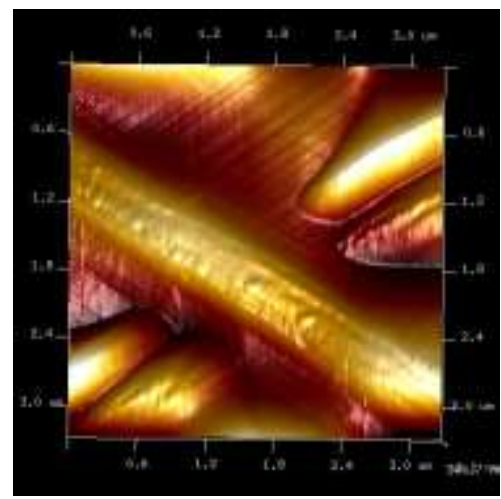


Fig13. AFM images of PVA/PAN nanofibers (a) unmodified (b) plasma modified 6min

\section{Conclusion}

In the current experiment, by examining the effect of exposing the PVA / PAN polymer nanofibers to air-DBD plasma during various times, has demonstrated that physico-chemical changes occurred at the nanofiber surface caused by interactions between the polymer surface and reactive species in plasma. According to ATR-FTIR spectra, the optimal plasma modification time of PVA / PAN nanofibers has been 6 minutes. Moreover, through spectrum analysis, it has been found that exposure to air plasma has increased the formation of $\mathrm{C}-\mathrm{O}, \mathrm{C}-\mathrm{H}$ and $\mathrm{C}=\mathrm{O}$ groups. Observation of increasing hydrophilicity in modified samples by measuring the water droplets contact angle demonstrated the effect of plasma on the hydrophilicity of nanofibers. The results of AFM image analysis have also been in line with this claim. The enhancement of active groups formed on the surface of PVA / PAN nanofibers by air-DBD plasma has demonstrated a significant effect on the immobilization rate of GOx enzyme, which has been affirmed by enzyme assay. Increasing the enzyme activity in the modified samples compared by unmodified samples has been a proof of this claim. In addition, augment in reusability and storage stability of enzyme have been investigated and reported in modified samples. Given that the results substantiate air-DBD plasma considered as an effective method in modifying PVA / PAN nanofibers, it could be asserted that this study has proposed a step towards producing biosensors based on enzyme immobilization. Furthermore, in clinical field, the GOx enzyme has played a momentous role.

\section{Acknowledgment}

The authors would like to express their gratitude to Staffs of Central Laboratory of Shahid Beheshti University, Tehran, Iran, for their help and patient in SEM imaging. We would also like to show our gratitude to the Fartash Khodaverdi (PhD student, Islamic Azad University) for sharing his wisdom with us during providing polymers for this research.

\section{Author contributions}

Saeed Javadi Anaghizi: Conceptualization, Methodology, Formal analysis, Investigation, Writing original draft. Sepideh Asadi: Methodology, Validation, Visualization. Hamid Ghomi: Investigation, Validation, Mehdi Jahanfar: Supervision, Writing - review \& editing, Resources.

\section{Data availability statement:}

The authors confirm that the data supporting the findings of this study are available within the article.

\section{References:}

[1]. F.-L. Zhou and R.-H. Gong, "Manufacturing technologies of polymeric nanofibers and nanofiber yarns,” Polymer International,vol.57, no.6,pp.837-845, (2008). 
[2]. A. Podg'orski, A. Bałazy, and L. Gradon', "Application of nanofi-bers to improve the filtration efficiency of the most penetrating aerosol particles in fibrous filters," Chemical Engineering Science,vol.61,no.20, pp.6804-6815, (2006).

[3]. N. Bhardwaj and S. C. Kundu, "Electrospinning: a fascinating fiber fabrication technique," Biotechnology Advances,vol.28, no. 3, pp. 325-347, (2010).

[4]. Woodhead Publishing Series in Textiles A2-Wei, Qufu, Func-tional Nanofibers and their Applications, Woodhead Publish-ing, pp. 15-19,(2012).

[5]. M. Langner and A. Greiner, "Wet-laid meets electrospinning: nonwovens for filtration applications from short electrospun polymer nanofiber dispersions," Macromolecular Rapid Com-munications,vol.37, no.4,pp.351-355, (2016).

[6]. S. Ouyang, T. Wang, X. Jia, Y. Chen, J. Yao, and S. Wang, "Self-indicating and recyclable superhydrophobic membranes for effective oil/water separation in harsh conditions," Materials \&Design,vol.96,pp.357-363, (2016).

[7]. C.-W. Li, Q. Wang, J. Li et al., "Silver nanoparticles/chitosan oligosaccharide/poly(vinyl alcohol) nanofiber promotes wound healing by activating TGF $\beta 1 /$ Smad signaling pathway," Interna-tional Journal of Nanomedicine, vol. 11, pp. 373-387, (2016).

[8]. Y. Mo, R. Guo, J. Liu et al., "Preparation and properties of PLGA nanofiber membranes reinforced with cellulose nanocrystals," Colloids and Surfaces B: Biointerfaces,vol.132,pp. 177-184, 2015 Y. Mo, R. Guo, J. Liu et al., "Preparation and properties of PLGA nanofiber membranes reinforced with cellulose nanocrystals," Colloids and Surfaces B: Biointerfaces,vol.132,pp. 177-184, (2015).

[9]. I. Yalcin, J. Horakova, P. Mikes, T. G. Sadikoglu, R. Domin, and D.Lukas, "Design of polycaprolactone vascular grafts," Journal of Industrial Textiles, vol. 45, no. 5, pp. 813-833, (2016).

[10]. R.L.Qi,R.Guo,M.W.Shenetal.,"Electrospunpoly(lactic-co-glycolic acid)/halloysite nanotube composite nanofibers for drug encapsulation and sustained release," Journal of Materials Chemistry,vol.20, no.47, pp.10622-10629, (2010).

[11]. K. Kalinova, "A sound absorptive element comprising an acoustic resonance nanofibrous membrane," Recent Patents on Nanotechnology,vol.9,no.1,pp.61-69, (2015).

[12]. L. Mares, J. Svobodova, and F. Yalcinkaya, "Method for applica-tion of a cosmetic composition containing at least one active substance to skin and a means for carrying out this method for application of the cosmetic composition," WO2016/050227, Nafigate Cosmetics, A.S. (Pod pek'arnami 245/10, Praha 9, 190 00, CZ), (2016).

[13]. J. Xu, L. Wang, J. Guan, and S. Yin, "Coupled effect of strain rate and solvent on dynamic mechanical behaviors of separators in lithium ion batteries," Materials \& Design, vol 95, pp.319-328, (2016). 
[14]. Y.Liao,R.Wang, M.Tian,C.Qiu,andA.G.Fane,"Fabrica-tion of polyvinylidene fluoride (PVDF) nanofiber membranes by electro-spinning for direct contact membrane distillation," Journal of Membrane Science,vol.425-426,pp. 30-39, (2013).

[15]. C. Feng,K.C.Khulbe, T. Matsuuraetal., "Productionofdrink-ing water from saline water by air-gap membrane distillation using polyvinylidene fluoride nanofiber membrane," Journal of Membrane Science, vol. 311, no. 1-2, pp. 1-6, (2008).

[16]. Z.M. Huang, Y.Z. Zhang, M. Kotaki, S. Ramakrishna, A review on polymer nanofibers by electrospinning and their applications in nanocomposites, Compos. Sci. Technol. 63 ,2223-2253, (2003).

[17]. J.D. Schiffman, C.L. Schauer, A review: electrospinning of biopolymer nanofibers and their applications, Polym. Rev. 48 ,317-352, (2008).

[18]. S. J. Cho, S. M. Jung, M. Kang, H. S. Shin, and J. H. Youk, "Preparation of hydrophilic PCL nanofiber scaffolds via electro-spinning of PCL/PVP-b-PCL block copolymers for enhanced cell biocompatibility," Polymer,vol.69, no.1,pp.95-102, (2015).

[19]. L. Zarybnicka, E. Stranska, J. Machotova, and G. Lencova, "Preparation of two-layer anionexchange poly(ethersulfone) based membrane: effect of surface modification," International Journal of Polymer Science,vol.2016, ArticleID8213694,8 pages, (2016).

[20]. N. Recek, M. Resnik, H. Motaln et al., "Cell adhesion on pol-ycaprolactone modified by plasma treatment," International Journal of Polymer Science,vol. 2016, Article ID7354396,9 pages, (2016).

[21]. J. Zhao,N.Dehbari,W.Han,L.Huang,and Y. Tang,"Elec-trospun multi-scale hybrid nanofiber/net with enhanced water swelling ability in rubber composites," Materials and Design,vol. 86, pp. 14-21, (2015).

[22]. S.Maretschek,A.Greiner,andT.Kissel, "Electrospunbi-odegradable nanofiber nonwovens for controlled release of proteins," Journal of Controlled Release,vol.127,no. 2, pp.180-187, (2008).

[23]. Liu, Y., et al. "Facile electrospun polyacrylonitrile/poly (acrylic acid) nanofibrous membranes for high efficiency particulate air filtration." Fibers and Polymers 16(3): 629-633, (2015).

[24]. F. Khodaverdi, A. Vaziri, M. Javanbakht, M. Jahanfar, Improvement of PAN separator properties using PVA/malonic acid by electrospinning in lithium ion-batteries, Vol,138, Issue13,50088,(2021).

[25]. A.A. Khan, M.A. Alzohairy, Recent advances and applications of immobilized enzyme technologies: a review, Res. J. Biol. Sci. 5 (8) ,565-575, (2010).

[26]. J. Kim, J.W. Grate, P. Wang, Nanostructures for enzyme stabilization, Chem. Eng. Sci. 61 (3) 10171026, (2006).

[27]. H. Liu, J. Qian, Y. Liu, T. Yu, J. Deng, Immobilization of glucose oxidase in the composite membrane of regenerated silk fibroin and poly(vinyl alcohol): application to an amperometric glucose sensor, Bioelectrochem. Bioenerg. 39 303-308, (1996). 
[28]. B. Unnikrishnan, S. Palanisamy, S.M. Chen, A simple electrochemical approach to fabricate a glucose biosensor based on graphene-glucose oxidase biocomposite, Biosens. Bioelectron. 39 (1) 70-75, (2013).

[29]. Wang, Y. and Y.-L. Hsieh "Immobilization of lipase enzyme in polyvinyl alcohol (PVA) nanofibrous membranes." Journal of Membrane Science 309(1-2): 73-81, (2008).

[30]. Wang, F., et al. "Reversible immobilization of glucoamylase by metal affinity adsorption on magnetic chelator particles." Journal of Molecular Catalysis B: Enzymatic 48(1-2): 1-7, (2007).

[31]. Bayramoğlu, G., et al. "Polyaniline grafted polyacylonitrile conductive composite fibers for reversible immobilization of enzymes: Stability and catalytic properties of invertase." Process Biochemistry 44(8): 880-885, (2009).

[32]. Y. Wan, J. Yang, J. Yang, J. Bei, S. Wang, Cell adhesion on gaseous plasma modified poly-(1lactide) surface under shear stress field, Biomaterials $24,3757-3764$, (2003).

[33]. J.Y. Wong, J.B. Leach, X.Q. Brown, Balance of chemistry, topography, and mechanics at the cellbiomaterial interface: issues and challenges for assessing the role of substrate mechanics on cell response, Surf. Sci. 570 119-133, (2004).

[34]. S. Han, Y. Lee, H. Kim, G. Kim, J. Lee, J.H. Yoon, G. Kim, Polymer surface modification by plasma source ion implantation, Surf. Coat. Technol. 93 ,261-264, (1997).

[35]. R. Nathawat, A. Kumar, N.K. Acharya, Y.K. Vijay, XPS and AFM surface study of PMMA irradiated by electron beam, Surf. Coat. Technol. 203 2600-2604,(2009).

[36]. A.S. Hoffman, Surface Modification of Polymers: Physical, Chemical, Mechanical and Biological Methods, Macromolecular Symposia, Wiley Online Library, pp. 443-454,( 1996).

[37]. M.V. Naseh, A.A. Khodadadi, Y. Mortazavi, F. Pourfayaz, O. Alizadeh, M. Maghrebi, Fast and clean functionalization of carbon nanotubes by dielectric barrier discharge plasma in air compared to acid treatment, Carbon 48 (5) 1369-1379, (2010).

[38]. F. Truica-Marasescu, S. Guimond, P. Jedrzejowski, M.R. Wertheimer, Hydrophobic recovery of VUV/NH 3 modified polyolefin surfaces: comparison with plasma treatments in nitrogen, Nucl. Instrum. Methods B $236,117-122$, (2005).

[39]. Y. Liu, C. Su, X. Ren, C. Fan, W. Zhou, F. Wang, W. Ding, Experimental study on surface modification of PET films under bipolar nanosecond-pulse dielectric barrier discharge in atmospheric air, Appl. Surf. Sci. 313 ,53-59, (2014).

[40]. J.M. Grace, L.J. Gerenser, Plasma treatment of polymers, J. Dispers. Sci. Technol. 24 305-341, (2003).

[41]. Anaghizi, S. J., et al. "The configuration effects of electrode on the performance of dielectric barrier discharge reactor for NO X removal." IEEE transactions on plasma science 43(6): 1944-1953, (2015). 
[42]. Blajan, M., et al. "Emission spectroscopy of microplasma driven by a pulsed power supply." J. Inst. Electrostat. Jpn. 34: 99-104, (2010).

[43]. Dorraki, N., et al. "Surface modification of chitosan/PEO nanofibers by air dielectric barrier discharge plasma for acetylcholinesterase immobilization." Applied Surface Science 349: 940-947, (2015).

[44]. Bouin, J. C., et al. "The glucose oxidase-catalase system. Methods in enzymology". Elsevier. 44: 478-488,( 1976).

[45]. Wu, S., et al. "Adhesion and adhesion reliability enhancement of evaporated copper on surface modified poly (tetrafluoroethylene) films from graft copolymerization." IEEE transactions on advanced packaging 23(3): 538-545, (2000).

[46]. Mirabedini, S., et al "Effect of low-pressure O2 and Ar plasma treatments on the wettability and morphology of biaxial-oriented polypropylene (BOPP) film." Progress in Organic Coatings 60(2): 105111, (2007).

[47]. Atkinson, R., et al., Evaluated Kinetic and Photochemical Data for Atmospheric Chemistry: Supplement VI. J. Phys. Chem. Ref, p. 1329-1499,( 1997).

[48]. BLAJAN, M., et al., Emission Spectroscopy of Microplasma Driven by a Pulsed Power Supply. J. Inst. Electrostat. Jpn.,34(2): p. 99-104,( 2010).

[49]. Kozlov, K.V. and H.-E. Wagner, Progress in Spectroscopic Diagnostics of Barrier Discharges. Plasma physics, 47(1): p. 26-33,( 2007). 